\title{
RUP'TURE OF THE UTERUS,
}

AND

SUBSEQUENT RECOVERY OF THE PATIENT.

By JAMES POWELL, Esq.

Read July 8, 1823.

IHAVE the honour of laying before the Society the following case of rupture of the uterus, and subsequent recovery of the patient, as the facts connected with it cannot fail to be interesting.

Ann Dance, aged 24, a patient in the parochial infirmary of St. Clement Danes, of low stature and considerable deformity of the bones of the limbs, and advanced to the full period of her first pregnancy, experienced some slight uterine pains on the evening of Monday, October 7, 1822. These pains continued lingering, with but little dilatation of the orifice of the uterus, until six o'clock on Thursday evening; at which time I found, upon examination, that the orifice of the uterus was dilated to the size of a crown piece, and that the head of the child presented.-Soon after this visit, very strong bearing down pains came on, which 
continued with increasing urgency until abput eight o'clock, when, according to the report of the midwife, (who had the patient under her immediate charge), these bearing pains suddenly and totally ceasing, were succeeded by an immediate, and a most excruciating pain of a different kind. This important change in the character of the labour, expressed, as it soon became, by great anxiety of countenance, and other indications of extreme distress, occasioned much alarm amongst the attendants, and my assistant, in consequence of my unavoidable absence from town, was requested forthwith to see the patient. That gentleman, in consideration of the intensity and permanence of the pain, was induced to have recourse to bleeding, and afterwards to administer an opiate.-At this time there was neither hemorrhage nor sickness present. On my return to town, about 11 o'clock, on the following day (Friday), I was convinced from her peculiarly distressed and anxious countenance, which forcibly struck me on entering her chamber, that something very serious had occurred in her case.-The abdomen presented, upon the whole, an uniform surface, similar to what it usually does in advanced pregnancy. On examination, per vaginam, however, I found that the head, which had before presented, as well as every other part of the child, had receded entirely beyond my reach. Presuming from the above circumstances, that rupture of the uterus had taken place, I concluded that instant delivery was the appropriate 
treatment, and I therefore lost no time in procuring the attendance of two or three of my professional brethren to sanction my decision. On the arrival of my friend Doctor Davis, about one o'clock, it was immediately determined to deliver the patient, that gentleman in the presence of Mr. Wray, Mr, Radnor, and myself, undertaking the operation.

The limbs of the child could not be felt through the abdominal parietes, as usually happens in these cases : but it, was observed by Doctor Davis, before the commencement of the operation, that the tumid abdomen presented two prominences, which, upon the introduction of thehand, he found were occasioned by the apposition of the back and head of the child toit: thelatter forming the inferior tumor, immediately above the brim of the pelvis, and the curved smooth back, the other-the hands and feet were folded as in ordinary cases, in front of the child, and, therefore, were of course directed towards the back of the mother. Doctor Davis ascertained that the rupture extended along the whole course of the neck of the womb, on the right side, including its orifice. He also reported, when his hand was in the cavity of the abdomen, that he felt the body of the uterus much contracted, and occupying posteriorly, towards the left side, the space immediately above the brim of the pelvis.-The delivery was effected by turning. The confinement of the pelvis at the brim (although not considerable) was such as to require the ope- 
ration of craniotomy, before the extraction of the head could be accomplished. So much was the strength of the patient exhausted at this time, that during the remainder of the operation, it became necessary to administer to her repeated table spoonsful of undiluted brandy to keep her in actual existence. The placenta, having been previously thrown off from the uterine surface, was found in the vagina, immediately after the removal of the child, and withdrawn by the application of the slightest traction. No hemorrhage, nor descent of any portion of intestine succeeded to this result. A full dose (60 minims) of Battley's Liquor Opii sedativus was immediately exhibited, and the same anodyne, in a smaller dose, was repeated at bed-time. On the following morning (the 12th) she was not only alive, contrary to the expectation of all the gentlemen who had witnessed her deplorable condition on the preceding day, but she was in many respects positively better : and although she had not absolutely slept during any part of the night, she had enjoyed a considerable degree of tranquillity. The pulse was 160 . The abdomen was tense, and extremely tender to the touch; the countenance was improved, but still anxious; the discharge from the vagina was sufficient, but of a dark grumous character. She passed her urine readily, and in proper quantity. The breathing was excessively laborious, and oppressed. Eight leeches were applied to the abdomen, and the bowels were opened by an enema. In the even- 
ing a full dose of opium was administered, and as soon as the wounds from the leeches had ceased to bleed, a large blister was applied to the hypogastrium. The same state of symptoms continued for several days ; the abdomen, however, becoming gradually less tense and less painful upon motion. After an interval of about eight days, pieces of organised structure, sloughy in their appearance, and very offensive from their putridity, escaped from the vagina. After this the uterine discharge assumed the character of laudable and healthy purulent matter. This new form of discharge continued for many days, but without being accompanied with any decided remission of the alarming symptoms; the excessive frequency of the pulse, the laborious respiration, the soreness of the abdomen, and the cough remaining unabated. The patient, however, began at this time to relish food, and she was allowed to indulge sparingly in the use of a light and nutrient diet, including a small portion, once daily, of animal food. The purulent discharge having ceased, a healthy evacuation of the Tochia followed. But this second change did not appear decisive of the patient's fate, inasmuch as several of her most urgent symptoms, such as extreme difficulty of breathing, incessant and violent coughing, and great pain of the hypogastrium upon attempting to change her position, continued greatly to harass her. In the midst of these unpromising circumstances, a large quantity of purulent matter was thrown up from 
the chest, in the midst of a fit of coughing, which had the effect of greatly relieving her most distressing symptoms. She continued to expectorate purulent matter for several days afterwards. Mild tonics, and a generous diet, with wine, which at this time were recommended, appeared to contribute importantly towards saving her life. Such, indeed, was her state of extreme exhaustion, until the purulent expectoration had completely ceased, that the exhibition of wine, in very frequent doses, was considered of indispensable necessity. When upon one occasion, from an idea that by its use the pulmonary affection was aggravated, the wine was partially discontinued, the symptoms of exhaustion became so suddenly formidable, as to compel an immediate return to its administration in the former quantities*. From this time her health gradually improved, and medicines were discontinued upon the $9 \mathrm{~d}$ of December. About the middle of the same month, she was discharged from the infirmary, quite well, without pain or cough, or any other affection. I have seen her several times since that period, and upon one occasion she complained of considerable tenderness of the lower part of the abdomen, which yielded to a moderate bleeding, and other appropriate treatment, and at present $I$ believe she is perfectly free from disease.

* The wine used upon this occasion was Sherry, and the quantity exhibited for unany days was a wine glass full every three or four hours. 